\title{
Least Squares Symmetrizable Solutions for a Class of Matrix Equations
}

\author{
Fanliang Li \\ School of Sciences, Institute of Mathematics and Physics, Central South University of \\ Forestry and Technology, Changsha, China \\ Email:1f1302@tom.com
}

Received January 30, 2013; revised March 27, 2013; accepted April 4, 2013

Copyright (C) 2013 Fanliang Li. This is an open access article distributed under the Creative Commons Attribution License, which permits unrestricted use, distribution, and reproduction in any medium, provided the original work is properly cited.

\begin{abstract}
In this paper, we discuss least squares symmetrizable solutions of matrix equations $(A X=B, X C=D)$ and its optimal approximation solution. With the matrix row stacking, Kronecker product and special relations between two linear subspaces are topological isomorphism, and we derive the general solutions of least squares problem. With the invariance of the Frobenius norm under orthogonal transformations, we obtain the unique solution of optimal approximation problem. In addition, we present an algorithm and numerical experiment to obtain the optimal approximation solution.
\end{abstract}

Keywords: Matrix Equations; Matrix Row Stacking; Topological Isomorphism; Least Squares Solution; Optimal Approximation

\section{Introduction}

The matrix equations $(A X=B, X C=D)$, where $A, B, C$, $D$ are usually given by experiments, have a long history [1]. Many authors considered these matrix equations. For example, Mitra [2,3], Chu [4] discussed its unconstraint solutions with the generalized inverse of matrix and the singular value decomposition (SVD), respectively. In recent years, many authors considered its constraint solutions. A series of meaningful results were achieved [1,5$10]$. The methods in these papers are mainly the generalized inverse of matrix and special properties of finite dimensional vector spaces $[1,5,6]$, the decomposition of matrix or matrix pairs $[7,8]$ and the special properties of constraint matrices $[9,10]$. However, the least squares symmetrizable solutions for these matrix equations have not been considered. The purpose of this paper is to discuss its least squares symmetrizable solutions with the matrix row stacking, Kronecker product and special relations between two linear subspaces which are topological isomorphism because the structure of symmetrizable matrices can not be found and the methods applied in [1-10] can not solve the problem in this paper. The background for introducing the definition of symmetrizable matrices is to get "symmetric" matrices from nonsymmetric matrices [11] because of nice properties and multi-areas of applications of symmetric matrices. For example, Sun [12] introduced the definition of positive definite sym- metrizable matrices to study an efficient algorithm for solving the nonsymmetry second-order elliptic discrete systems.

Throughout this paper we use some notations as follows. Let $R^{n \times m}$ be the set of all $n \times m$ real matrices and denote $R^{n}=R^{n \times 1} ; O R^{n \times n}, S R^{n \times n}$ and $A S R^{n \times n}$ are the set of all $n \times n$ orthogonal, symmetric and skewsymmetric matrices, respectively. $R(A), A^{\mathrm{T}}$ and $A^{+}$ represent the range, the transpose and the Moore-Penrose generalized inverse of $A$, respectively.

$I_{n}$ denotes the identity matrix of order $n$. For $A=\left(a_{i j}\right)$, $B=\left(b_{i j}\right) \in R^{n \times m}, A \otimes B$ denotes Kronecker product of matrix $A$ and $B ;\langle A, B\rangle=\operatorname{tr}\left(B^{\mathrm{T}} A\right)$ denotes the inner product of matrix $A$ and $B$. The induced matrix norm is called Frobenius norm, i.e. $\|A\|=\langle A, A\rangle^{1 / 2}$, then $R^{n \times m}$ is a Hilbert inner product space.

Definition 1. A real $n \times n$ matrix $A$ is called a symmetrizable (skew-symmetrizable) matrix if $A$ is similar to a symmetric (skew-symmetric) matrix $A$. The set of symmetrizable (skew-symmetrizable) matrices is denoted by $S Z R^{n \times n} \quad\left(A S Z R^{n \times n}\right)$.

From Definition 1, it is easy to prove that $A \in S Z R^{n \times n}$ $\left(A S Z R^{n \times n}\right)$ if and only if there exists a nonsingular matrix $W$ and a symmetric (skew-symmetric) matrix $\bar{A}$ such that

$$
A=W \bar{A} W^{-1}
$$

We now introduce the following two special classes of 
subspaces in $R^{n \times n}$.

$$
\begin{gathered}
S R^{n \times n}(W)=\left\{A \mid A=W \bar{A} W^{-1}, \forall A \in S R^{n \times n}\right\}, \\
A S R^{n \times n}(W)=\left\{A \mid A=W \bar{A} W^{-1}, \forall A \in A S R^{n \times n}\right\} .
\end{gathered}
$$

It is easy to see that if $W$ is a given nonsingular matrix, then $S R^{n \times n}(W)$ and $A S R^{n \times n}(W)$ are two closed linear subspace of $R^{n \times n}$. In this paper, we suppose that $W$ is a given nonsingular matrix and $W \neq I_{n}$. We will consider the following problems.

Problem I. Giving $A, B \in R^{h \times n}, C, D \in R^{n \times l}$, find $X \in S R^{n \times n}(W)$ such that

$$
\min \left(\|A X-B\|^{2}+\|X C-D\|^{2}\right) .
$$

Problem II. Given $X^{*} \in R^{n \times n}$, find $\hat{X} \in S_{E}$ such that

$$
\left\|X^{*}-\hat{X}\right\|=\min _{\forall X \in S_{E}}\left\|X^{*}-X\right\|,
$$

where $S_{E}$ is the solution set of Problem I.

In this paper, if $C=0, D=0$ in Problem I, then Problem I becomes Problem I of [13]. Peng [13] studied the least squares symmetrizable solutions of the matrix equation $A X=B$ with the singular value decomposition of matrix. The method applied in [13] can not solve Problem $\mathrm{I}$ in this paper. In this paper, we first take matrix equations $(A X=B, X C=D)$ into linear equations with matrix row stacking and Kronecker product. Then we obtain an orthogonal basis-set for $S R^{n \times n}(W)$ with special relations between two linear subspaces which are topological isomorphism. Based on these results, we obtain the general expression of Problem I.

This paper is organized as follows. In Section 2, we first discuss the matrix row stacking methods, Kronecker product of matrix and relations between $S R^{n \times n}(W)$ and $S R^{n \times n}$. Then we obtain the general solutions of Problem I. In Section 3, we derive the solution of Problem II with the invariance of Frobenius norm under orthogonal transformations. In the end, we give an algorithm and numerical experiment to obtain the optimal approximation solution.

\section{The Solution Set of Problem I}

At first, we discuss the matrix row stacking methods, Kronecker product of matrix and relations between two linear subspaces which are topological isomorphism.

For any $A \in R^{m \times n}$, let $\operatorname{vec}(A)$ denote an ordered stack of the row of $A$ from upper to low stacking with the first row, i.e.

$$
\operatorname{vec}(A)=[A(1:), A(2:), \cdots, A(m:)]^{\mathrm{T}} \in R^{m n},
$$

where $A(i:)$ denotes the $i$ th row of $A$. For any vector $x \in R^{m n}$, let $\operatorname{vec}^{-1}(x)$ denote the following matrix containing all the entries of vector $x$.

$$
\operatorname{vec}^{-1}(x)=\left(\begin{array}{c}
x(1: n)^{\mathrm{T}} \\
x(n+1: 2 n)^{\mathrm{T}} \\
\vdots \\
x(m(n-1): m n)^{\mathrm{T}}
\end{array}\right) \in R^{m \times n},
$$

where $x(i: j)$ denotes the elements from $i$ to $j$ of vector $x$. From (2.1), we can derive the following two linear subspaces of $R^{n^{2}}$.

$$
\begin{gathered}
\operatorname{vec}\left(S R^{n \times n}\right)=\left\{\operatorname{vec}(A) \mid A \in S R^{n \times n}\right\}, \\
\operatorname{vec}\left(S R^{n \times n}(W)\right)=\left\{\operatorname{vec}(A) \mid A \in S R^{n \times n}(W)\right\} .
\end{gathered}
$$

Lemma 1. [14] If $A \in R^{h \times m}, B \in R^{n \times l}, X \in R^{m \times n}$, then

$$
\operatorname{vec}(A X B)=\left(A \otimes B^{\mathrm{T}}\right) \operatorname{vec}(X) .
$$

For any $\bar{X} \in S R^{n \times n}$, let

$$
f^{\prime}: \bar{X} \rightarrow W \bar{X} W^{-1} \text {, i.e. } f^{\prime}(\bar{X})=W \bar{X} W^{-1} .
$$

It is no difficult to prove that mapping $f^{\prime}$ is a topological isomorphism mapping from $S R^{n \times n}$ to $S R^{n \times n}(W)$. According to (2.1) and (2.5), it is easy to derive the following mapping from linear subspaces $\operatorname{vec}\left(S R^{n \times n}\right)$ to $\operatorname{vec}\left(S R^{n \times n}(W)\right)$.

$$
f: \operatorname{vec}(\bar{X}) \rightarrow\left(W \otimes W^{-T}\right) \operatorname{vec}(\bar{X}),
$$

i.e.

$$
f(\operatorname{vec}(\bar{X}))=\left(W \otimes W^{-T}\right) \operatorname{vec}(\bar{X})
$$

It is also easy to prove that mapping $f$ is a topological isomorphism mapping from $\operatorname{vec}\left(S R^{n \times n}\right)$ to $\operatorname{vec}\left(S R^{n \times n}(W)\right)$. It is clear that the dimension of $S R^{n \times n}$ is $\frac{n(n+1)}{2}$. This implies that the dimension of $\operatorname{vec}\left(S R^{n \times n}\right)$ and $\operatorname{vec}\left(S R^{n \times n}(W)\right)$ are also $\frac{n(n+1)}{2}$. In this paper, let $k=\frac{n(n+1)}{2}$.

Lemma 2. If $\alpha_{1}, \alpha_{2}, \cdots, \alpha_{k}$ is an orthonormal basisset for $\operatorname{vec}\left(\operatorname{SR}^{n \times n}(W)\right)$, and let $\Lambda=\left(\alpha_{1}, \alpha_{2}, \cdots, \alpha_{k}\right)$, then the following relations hold.

$$
\begin{gathered}
\Lambda \in R^{n^{2} \times k}, \Lambda^{\mathrm{T}} \Lambda=I_{k}, \\
R(\Lambda)=\operatorname{vec}\left(S R^{n \times n}(W)\right), R\left(\Lambda^{\mathrm{T}}\right)=R^{k} .
\end{gathered}
$$

From the definition of the orthonormal basis-set, it is easy to prove Lemma 2, so the proof is omitted.

For any matrix $A \in S R^{n \times n}(W)$, if let $v e \tilde{c}(A) \in R^{k}$ denote the vector of coordinates of $\operatorname{vec}(A)$ with respect to the basis-set $\alpha_{1}, \alpha_{2}, \cdots, \alpha_{k}$, then combining (2.4) and 
(2.7), we have

$$
\begin{aligned}
& \operatorname{vec}(A)=\Lambda \operatorname{ve} \tilde{c}(A) \in R^{n^{2}}, \\
& \operatorname{vec}(A)=\Lambda^{\mathrm{T}} \operatorname{vec}(A) \in R^{k} .
\end{aligned}
$$

Moreover, for any $x \in R^{k}$, the following conclusion holds.

$$
\operatorname{vec}^{-1}(\Lambda x) \in S R^{n \times n}(W) .
$$

The orthonormal basis-set $\alpha_{1}, \alpha_{2}, \cdots, \alpha_{k}$ for $\operatorname{vec}\left(S R^{n \times n}(W)\right)$ can be obtained by the following calculation procedure.

\section{Calculation procedure}

Step 1. Input a basis-set $A_{1}, A_{2}, \cdots, A_{k}$ for $S R^{n \times n}$.

Step 2. According to (2.1), compute $\bar{\alpha}_{i}=\operatorname{vec}\left(A_{i}\right)$, $i=1,2, \cdots, k$, and obtain a basis-set $\bar{\alpha}_{1}, \bar{\alpha}_{2}, \cdots, \bar{\alpha}_{k}$ for $\operatorname{vec}\left(S R^{n \times n}\right)$.

Step 3. Input a nonsingular matrix $W$, compute

$$
\left(W \otimes W^{-T}\right) \bar{\alpha}_{1},\left(W \otimes W^{-T}\right) \bar{\alpha}_{2}, \cdots,\left(W \otimes W^{-T}\right) \bar{\alpha}_{k},
$$

and obtain a basis-set for $\operatorname{vec}\left(S R^{n \times n}(W)\right)$.

Step 4. Compute the $\mathrm{QR}$ decomposition of matrix

$$
\left(W \otimes W^{-T}\right) \bar{\alpha}_{1},\left(W \otimes W^{-T}\right) \bar{\alpha}_{2}, \cdots,\left(W \otimes W^{-T}\right) \bar{\alpha}_{k},
$$

and obtain an orthonormal basis-set $\alpha_{1}, \alpha_{2}, \cdots, \alpha_{k}$ for $\operatorname{vec}\left(S R^{n \times n}(W)\right)$.

Lemma 3. If $A \in R^{h \times m}, B \in R^{h \times n}$, then the general solutions of least squares problem

is

$$
\min _{X \in R^{m \times n}}\|A X-B\|
$$

$$
X=A^{+} B+\left(I_{m}-A^{+} A\right) K, \forall K \in R^{m \times n} .
$$

With the singular value decomposition of matrix, it is easy to prove this lemma. So the proof is omitted.

Theorem 1. Giving $A, B \in R^{h \times n}, C, D \in R^{n \times l}$, and let

$$
A_{0}=\left(\begin{array}{c}
A \otimes I_{n} \\
I_{n} \otimes C^{\mathrm{T}}
\end{array}\right) \Lambda, B_{0}=\left(\begin{array}{c}
\operatorname{vec}(B) \\
\operatorname{vec}(D)
\end{array}\right),
$$

then the general solutions of Problem $\mathrm{I}$ is

$$
\begin{aligned}
& X=\operatorname{vec}^{-1}(\Lambda \alpha), \\
& \alpha=A_{0}^{+} B_{0}+\left(I_{k}-A_{0}^{+} A_{0}\right) K, \forall K \in R^{k} .
\end{aligned}
$$

Proof. From Lemma 1, we have

$$
\begin{aligned}
&\|A X-B\|^{2}+\|X C-D\|^{2} \\
&=\left\|\left(A \otimes I_{n}\right) \operatorname{vec}(X)-\operatorname{vec}(B)\right\|^{2} \\
&+\left\|\left(I_{n} \otimes C^{\mathrm{T}}\right) \operatorname{vec}(X)-\operatorname{vec}(D)\right\|^{2} \\
&=\left\|\left(\begin{array}{c}
A \otimes I_{n} \\
I_{n} \otimes C^{T}
\end{array}\right) \operatorname{vec}(X)-\left(\begin{array}{c}
\operatorname{vec}(B) \\
\operatorname{vec}(D)
\end{array}\right)\right\|^{2} .
\end{aligned}
$$

This implies that finding $X \in S R^{n \times n}(W)$ such that $\min \left(\|A X-B\|^{2}+\|X C-D\|^{2}\right)$ if and only if finding $\alpha \in R^{k}$ such that

$$
\min \left\|\left(\begin{array}{c}
A \otimes I_{n} \\
I_{n} \otimes C^{\mathrm{T}}
\end{array}\right) \Lambda \alpha-\left(\begin{array}{c}
\operatorname{vec}(B) \\
\operatorname{vec}(D)
\end{array}\right)\right\|^{2},
$$

where $\Lambda \alpha=\operatorname{vec}(X)$. From Lemma 3, the general solutions of (2.13) is

$$
\alpha=A_{0}^{+} B_{0}+\left(I_{k}-A_{0}^{+} A_{0}\right) K, \forall K \in R^{k} .
$$

Combining (2.13) and (2.14) gives (2.12).

\section{The Solution of Problem II}

Let $S_{E}$ be the solution set of Problem I. From (2.12), it is easy to see that $S_{E}$ is a nonempty closed convex set. So we claim that for any given $X^{*} \in R^{n \times n}$, there exists the unique optimal approximation for Problem II.

Theorem 2. If given $A, B \in R^{h \times n}, C, D \in R^{n \times l}, X^{*} \in$ $R^{n \times n}$, then Problem II has a unique solution $\hat{X} \in S_{E}$. Moreover, $\hat{X}$ can be expressed as

$$
\begin{aligned}
& \hat{X}=\operatorname{vec}^{-1}(\Lambda \hat{\alpha}), \\
& \hat{\alpha}=A_{0}^{+} B_{0}+\left(I_{k}-A_{0}^{+} A_{0}\right) \Lambda^{\mathrm{T}} \operatorname{vec}(X),
\end{aligned}
$$

where $A_{0}, B_{0}$ are denoted by (2.11)

Proof. Choose $\tilde{\Lambda}$ such that $(\Lambda, \tilde{\Lambda}) \in O R^{n^{2} \times n^{2}}$. Combining the invariance of the Frobenius norm under orthogonal transformations, (2.12) and (2.14), we have

$$
\begin{aligned}
\left\|X^{*}-X\right\|^{2}= & \left\|\operatorname{vec}\left(X^{*}\right)-\operatorname{vec}(X)\right\|^{2} \\
& =\left\|\operatorname{vec}\left(X^{*}\right)-\Lambda \alpha\right\|^{2} \\
& =\left\|\left(\begin{array}{c}
\Lambda^{\mathrm{T}} \\
\tilde{\Lambda}^{\mathrm{T}}
\end{array}\right) \operatorname{vec}\left(X^{*}\right)-\left(\begin{array}{c}
\Lambda^{\mathrm{T}} \\
\tilde{\Lambda}^{\mathrm{T}}
\end{array}\right) \Lambda \alpha\right\|^{2} \\
= & \left\|\Lambda^{\mathrm{T}} \operatorname{vec}\left(X^{*}\right)-A_{0}^{+} B_{0}-\left(I_{n}-A_{0}^{+} A_{0}\right) K\right\|^{2} \\
& +\left\|\tilde{\Lambda}^{\mathrm{T}} \operatorname{vec}\left(X^{*}\right)\right\| .
\end{aligned}
$$

Let $E=I_{k}-A_{0}^{+} A_{0}, E_{1}=I_{k}-E$, it is clear that $E, E_{1}$ are orthogonal projection matrices satisfying $E E_{1}=0$. Hence, we have

$$
\begin{aligned}
\left\|X^{*}-X\right\|^{2}= & \left\|E\left(\Lambda^{\mathrm{T}} \operatorname{vec}\left(X^{*}\right)-A_{0}^{+} B_{0}-K\right)\right\|^{2} \\
& +\left\|E_{1}\left(\Lambda^{\mathrm{T}} \operatorname{vec}\left(X^{*}\right)-A_{0}^{+} B_{0}\right)\right\|^{2} \\
& +\left\|\tilde{\Lambda}^{\mathrm{T}} \operatorname{vec}\left(X^{*}\right)\right\| .
\end{aligned}
$$

It is easy to prove that $E A_{0}^{+} B_{0}=0$. This implies that

$$
\begin{aligned}
& \min _{\forall X \in S_{E}}\left\|X^{*}-X\right\| \\
& \Leftrightarrow \min _{\forall K \in R^{k}}\left\|E\left(\Lambda^{\mathrm{T}} \operatorname{vec}\left(X^{*}\right)-K\right)\right\| .
\end{aligned}
$$


The solution of (3.2) is

$$
K=\Lambda^{\mathrm{T}} \operatorname{vec}\left(X^{*}\right)+E_{1} K_{1}, \forall K_{1} \in R^{k} .
$$

Substituting (3.3) to (2.12) gives (3.1).

From Theorem 2, we can design the following algorithm to obtain the optimal approximate solution.

\section{Algorithm}

1) Input $A, B, C, D, W, X^{*}$.

2) Input a basis-set $A_{1}, A_{2}, \cdots, A_{k}$ for $S R^{n \times n}$.

$3)$ According to the calculation procedure before, compute $\alpha_{1}, \alpha_{2}, \cdots, \alpha_{k}$ and obtain an orthonormal basis-set for $\operatorname{vec}\left(S R^{n \times n}(W)\right)$

4) Let $\Lambda=\alpha_{1}, \alpha_{2}, \cdots, \alpha_{k}$, compute $A_{0}, B_{0}$ from (2.11).

5) Compute $\hat{\alpha}$ from the second equation of (3.1). $\hat{X}$.

6) According to the first equation of (3.1), calculate

Example $(n=3, h=4, l=5)$

1) Input $A, B, C, D, W, X^{*}$ as follows.

$$
\begin{aligned}
& A=\left(\begin{array}{ccc}
1.9 & 5.4 & 6.5 \\
2.8 & 7.8 & 3.9 \\
3.6 & -0.9 & 7.5 \\
-2.7 & 2.1 & -3.9
\end{array}\right), \\
& B=\left(\begin{array}{ccc}
5.9 & 12.3 & -7.5 \\
10.3 & 4.1 & 2.9 \\
-3.7 & 5.6 & 3.7 \\
5.1 & 1.3 & -8.5
\end{array}\right), \\
& C=\left(\begin{array}{ccccc}
4.1 & 5.6 & 5.4 & 3.1 & 1.6 \\
3.2 & -1.9 & 7.6 & 7.8 & 3.9 \\
1.9 & 2.8 & 4.3 & -2.3 & 2.7
\end{array}\right), \\
& D=\left(\begin{array}{ccccc}
-1.3 & 7.6 & 6.7 & -2.1 & 2.9 \\
5.9 & 2.3 & 1.8 & 3.1 & 5.6 \\
4.5 & 5.4 & 6.7 & 5.7 & 7.3
\end{array}\right) \text {, } \\
& W=\left(\begin{array}{lll}
1 & 2 & 3 \\
2 & 2 & 1 \\
3 & 4 & 3
\end{array}\right) \\
& X^{*}=\left(\begin{array}{ccc}
5.8 & 1.7 & 2.9 \\
3.9 & -2.5 & 8.5 \\
-2.4 & 3.3 & 7.6
\end{array}\right)
\end{aligned}
$$

2) Input a basis-set $A_{1}, A_{2}, \cdots, A_{6}$ for $S R^{3 \times 3}$ as follows.

$$
A_{1}=\left(\begin{array}{lll}
1 & 0 & 0 \\
0 & 0 & 0 \\
0 & 0 & 0
\end{array}\right), A_{2}=\left(\begin{array}{lll}
0 & 1 & 0 \\
1 & 0 & 0 \\
0 & 0 & 0
\end{array}\right), A_{3}=\left(\begin{array}{lll}
0 & 0 & 1 \\
0 & 0 & 0 \\
1 & 0 & 0
\end{array}\right),
$$

$$
A_{4}=\left(\begin{array}{lll}
0 & 0 & 0 \\
0 & 1 & 0 \\
0 & 0 & 0
\end{array}\right), A_{5}=\left(\begin{array}{lll}
0 & 0 & 0 \\
0 & 0 & 1 \\
0 & 1 & 0
\end{array}\right), A_{6}=\left(\begin{array}{lll}
0 & 0 & 0 \\
0 & 0 & 0 \\
0 & 0 & 1
\end{array}\right) .
$$

3) According to the calculation procedure, we obtain an orthonormal basis-set $\alpha_{1}, \alpha_{2}, \cdots, \alpha_{6}$ for $\operatorname{vec}\left(S R^{3 \times 3}(W)\right)$ as follows.

$$
\begin{gathered}
\alpha_{1}=\left(\begin{array}{c}
-0.0714 \\
-0.2143 \\
0.1429 \\
-0.1429 \\
-0.4286 \\
0.2857 \\
-0.2143 \\
-0.6429 \\
0.4286
\end{array}\right), \alpha_{2}=\left(\begin{array}{c}
-0.0785 \\
-0.6021 \\
0.2792 \\
0.3316 \\
0.2618 \\
-0.4189 \\
0.2531 \\
-0.3403 \\
-0.1396
\end{array}\right), \alpha_{3}=\left(\begin{array}{c}
0.3143 \\
0.2119 \\
-0.2280 \\
0.3475 \\
-0.4198 \\
0.1065 \\
0.6618 \\
-0.2079 \\
-0.1215
\end{array}\right), \\
\alpha_{4}=\left(\begin{array}{c}
-0.1556 \\
-0.4003 \\
-0.0976 \\
-0.3984 \\
-0.3584 \\
-0.2075 \\
-0.2524 \\
-0.2674 \\
-0.3051 \\
-0.6509
\end{array}\right), \alpha_{5}=\left(\begin{array}{c}
-0.8084 \\
0.3081 \\
0.3880 \\
-0.2461 \\
-0.4215 \\
0.3096 \\
0.2170 \\
0.4156 \\
-0.3334
\end{array}\right), \alpha_{6}=\left(\begin{array}{c}
0.0050 \\
0.3604 \\
-0.2590 \\
-0.1224 \\
-0.0942 \\
-0.0535 \\
-0.1670
\end{array}\right)
\end{gathered}
$$

4) Using the software "MATLAB", we obtain the unique solution $\hat{X}$ of Problem II.

$$
\hat{X}=\left(\begin{array}{ccc}
-0.3657 & 0.1512 & 0.5837 \\
0.6314 & 0.4846 & -0.2603 \\
0.5852 & 0.6459 & 0.0550
\end{array}\right) \text {. }
$$

\section{Conclusion}

In this paper, we first derive the least squares symmetrizable solutions of matrix equations $(A X=B, X C=D)$ with the matrix row stacking and the theory of topological isomorphism, i.e. Theorem 1. Then we give the unique optimal approximation solution, i.e. Theorem 2. Based on Theorem 1 and 2, we design an algorithm to find the optimal approximation solution. Compare to [1-10], this paper has two important achievements. One is we apply the topological isomorphism theory to obtain the least squares symmetrizable solutions of matrix equations $(A X$ $=B, X C=D$ ), and provide a method to solve the matrix equation, where the construct of constraint matrix can not be found. The other is we present a stable calculation procedure to obtain an orthonormal basis-set for 
$\operatorname{vec}\left(S R^{n \times n}(W)\right)$, and solve the key problem of the algorithm.

\section{Acknowledgements}

The authors are very grateful to the referee for their valuable comments, and also thank for his helpful suggestions.

This research was supported by National natural Science Foundation of China (31170532).

\section{REFERENCES}

[1] A. Dajić and J. J. Koliha, "Equations $a x=c$ and $x b=b$ in Rings and Rings with Involution with Applications to Hilbert Space Operators," Linear Algebra and Its Applications, Vol. 429, No. 7, 2008, pp. 1779-1809. doi:10.1016/j.laa.2008.05.012

[2] S. K. Mitra, "The Matrix Equations $A X=C, X B=D$," Linear Algebra and Its Applications, Vol. 59, 1984, pp. 171-181. doi:10.1016/0024-3795(84)90166-6

[3] K. W. E. Chu, "Singular Value and Generalized Singular Value Decomposition and the Solution of Linear Matrix Equations," Linear Algebra and Its Applications, Vol. 88-89, 1987, pp. 83-98. doi:10.1016/0024-3795(87)90104-2

[4] S. K. Mitra, "A Pair of Simultaneous Linear Matrix Equations $A_{1} X B_{1}=C_{1}, A_{2} X B_{2}=C_{2}$ and a Matrix Programming Problem," Linear Algebra and Its Applications, Vol. 131, 1990, pp. 107-123. doi:10.1016/0024-3795(90)90377-O

[5] A. Dajić and J. J. Koliha, "Positive Solutions to the Equations $A X=C$, and $X B=D$ for Hilbert Space Operators," Journal of Mathematical Analysis and Applications, Vol. 333, No. 2, 2007, pp. 567-576. doi:10.1016/i.jmaa.2006.11.016
[6] Q. X. Xu, "Common Hermitian and Positive Solutions to the Adjointable Operator Equations $A X=C, X B=D$," Linear Algebra and Its Applications, Vol. 429, No. 1, 2008, pp. 1-11. doi:10.1016/j.laa.2008.01.030

[7] Q. W. Wang, "Bisymmetric and Centrosymmetric Solutions to Systems of Real Quaternion Matrix Equations," Computers and Mathematics with Applications, Vol. 49, No. 5-6, 2005, pp. 641-650. doi:10.1016/j.camwa.2005.01.014

[8] Y. Qiu and A. Wang, "Least Squares Solutions to the Equations $A X=B, X C=D$ with Some Constraints," Applied Mathematics and Computation, Vol. 204, No. 2, 2008, pp. 872-880. doi:10.1016/j.amc.2008.07.035

[9] F. L. Li, X. Y. Hu and L. Zhang, "The Generalized Reflexive Solution for a Class of Matrix Equations $(A X=B$, $X C=D)$," Acta Mathematica Scientia Series B, Vol. 1, No. 28, 2008, pp. 185-193.

[10] F. L. Li, X. Y. Hu and L. Zhang, "The Generalized Anti-Reflexive Solution for a Class of Matrix Equations $(B X=C, X D=E)$," Computational \& Applied Mathematics, Vol. 1, No. 27, 2008, pp. 31-46.

[11] O. Taussky, "The Role of Symmetric Matrices in the Study of General Matrices," Linear Algebra and Its Applications, Vol. 51, 1972, pp. 13-18.

[12] S. J. Chang, "On Positive Symmetrizable Matrices and Pre-Symmetry Iteration Algorithms," Mathematica $\mathrm{Nu}$ merica Sinica, Vol. 3, No. 22, 2000, pp. 379-384.

[13] Z. Y. Peng, "The Least-Squares Solution of Inverse Problem for One Kind of Symmetrizable Matrices," Chinese Journal of Numerical Mathematics and Applications, No. 3, 2004, pp. 219-224.

[14] D. W. Fausett and C. T. Fulton, "Large Least Squares Problems Involving Kronecker Products," SIAM Journal on Matrix Analysis and Applications, Vol. 15, No. 1, 1994, pp. 219-227. doi:10.1137/S0895479891222106 